\title{
¿ES INDISOLUBLE LA UNIDAD ENTRE RAZÓN Y DOMINIO? LA CRÍTICA DE LA RAZÓN EN HORKHEIMER Y HABERMAS
}

\author{
EDUARDO CONTRERAS \\ IES Salvador Serrano, \\ Alcaudete (Jaén)
}

\begin{abstract}
RESUMEN: El objetivo del presente artículo consiste en cuestionarnos si la ecuación entre razón y dominio pertenece a la esencia de la razón y por ello, su misma autorreflexión crítica sería autocontradictoria, o si, por el contrario, siendo una situación histórica o epocal, son posibles sus fundamentos normativos. Para su esclarecimiento confrontaremos las obras de Horkheimer Crítica de la razón instrumental ${ }^{1}$, Dialéctica de la llustración ${ }^{2}$ y Razón y Autoconservación ${ }^{3}$ con el replanteamiento de Habermas en Conocimiento e Interés ${ }^{4}$.
\end{abstract}

PALABRAS CLAVE: razón, interés, dominio, emancipación, Habermas, Horkheimer.

\section{Is Unity between Reason and Domain Indissoluble? Critique of Reason according to Horkheimer and Habermas}

\begin{abstract}
The aim of this article is to consider whether the equation between reason and domain belongs to the essence of reason, and consequently, its own critical self-reflection would be selfcontradictory, or if on the contrary, being this a historical situation, its regulatory fundamentals are possible. To clarify this, we are going to confront the works of Horkheimer Critique of Instrumental Reason, Dialectic of Enlightenment and Reason and Self-preservation to the rethinking of the question made by Habermas in his work Knowledge and Human Interests.
\end{abstract}

KEY WORDS: reason, interest, domain, emancipation, Habermas, Horkheimer.

1 Horkheimer. M., Zur Kritik der instrumentellen Vernunft, Frankfurt am Main, Athenäum Fischer Taschenbuch Verlag, 1974. Hay traducción castellana: Crítica de la razón instrumental. Madrid: Trotta, 2002. (Traducido por Juan José Sánchez). Apareció en el 47 bajo el título El eclipse de la razón y ya el 67 con el título de la presente edición. Su contenido y el de las dos anteriores es idéntico, el cual coincide con el proyecto original del Horkheimer.

2 Horkheimer, M., Adorno, Th. W., Dialektik der Aufklärung, Frankfurt am Main, Fischer Taschenbuch Verlag, 1978. Hay traducción castellana: Dialéctica de la Ilustración. Madrid: Trotta, 2009. (Traducido por Juan José Sánchez). Apareció por primera vez en el año 44 bajo el título Fragmentos Filosóficos y ya en el 47 como Dialéctica de la Ilustración. Corresponden a la edición del 44 los siguientes apartados de la edición actual: Concepto de Ilustración, Excursus I, Excursus II y La Industria cultural: La Ilustración como engaño de masas. Los apartados de la edición actual Apuntes y Esbozos, Elementos de antisemitismo aparecieron por primera vez en el 46 y se incluyeron en la edición del 47.

3 Horkheimer, M., Vernunft und Selbsterhaltung, Frankfurt am Main, S. Fischer Verlag, 1970. Hay traducción castellana: Teoría Crítica, Barcelona, Barral, 1971. (Traducido por Juan J. del Solar B.). No se trata de una obra propiamente dicha de Horkheimer, sino de una colección de artículos aparecidos entre el 1940 y el 1971. El artículo que nos ocupa, Razón y Autoconservación, fue publicado por primera vez en 1942.

4 Habermas, J., Erkenntnis und Interesse, Frankfurt am Main, Suhrkamp Verlag, 1968. Hay traducción castellana: Conocimiento e Interés. Madrid: Taurus, 1982. (Traducido por José Vidal Beneyto) 


\section{La RAZÓn COMO INSTRUMENTO DE DOMINIO}

\subsection{La unidad esencial y originaria entre razón y dominio en Horkheimer.}

En la DI ${ }^{5}$ se defiende como tesis general la unidad sustancial y originaria entre razón y dominio. Esto excluye de la razón su orientación a la verdad, ya que la versión instrumental de la razón, para ejercer su dominio omnímodamente, liquida toda presuposición que pretenda ser en sí misma racional y verdadera. Por lo tanto, si no es posible una razón crítica que se distancie respecto de los hechos, si no hay una utopía oculta en el concepto de razón, la misma DI incurre en contradicción consigo misma. Con esta aporía, descubierta en la DI, se muestra la situación trágica de una época histórica en la que la razón no funciona pero, dado que «no albergamos la menor duda de que la libertad en la sociedad es inseparable del pensamiento ilustrado» ${ }^{6}$, tampoco caben opciones irracionales ${ }^{7}$.

Para Horkheimer y Adorno, si la razón es esencialmente instrumento de domino, se obtienen tres consecuencias fundamentales ${ }^{8}$ :

- Si la ciencia empírica, estrictamente orientada al dominio de la naturaleza, constituye el «círculo mágico $»^{9}$ del verdadero conocimiento, entonces aquella queda reducida a sustrato de dominio universal.

- En cuanto que el dominio ejercido por las ciencias para disponer plenamente de la naturaleza supone un sujeto que se posee totalmente a sí mismo y que además se opone, se distancia y se afirma contra el contexto natural, la misma lógica del dominio se vuelve contra él. La negación de sí mismo como viviente natural oscurece y liquida completamente los fines para el dominio exterior, lo cual convierte en irracional toda acción de la razón instrumental. El sujeto en plena disposición de sí mismo y que ejerce dominio sobre lo otro, al marcar una relación unidireccional entre el sí mismo que confiere sentido y el objeto privado de éste, se desvincula de la realidad y se declara omnipotente respecto a ella, lo que acaba por liquidar los fines mismos del dominio.

5 Las abreviaturas de las obras serán las siguientes: Crítica de la razón instrumental será CrI en castellano y KiV en alemán, Dialéctica de la Ilustración será DI en castellano y DA en alemán, Razón y Autoconservación será RyA en castellano y VuS en alemán y Conocimiento e Interés será CI y EuI en alemán. En las citas se indicarán las siglas y las páginas separadas por una barra en ambos idiomas.

6 Cfr. Horkheimer, M., Adorno, Th. W., DI, p. 53/DA, p. 3.

7 Cfr. Perlini, T., La Escuela de Frankfurt. Caracas: Ed. Monte Ávila, 1976 (Traducido por Margara Russotto). Consultar, pp. 85-98, el epígrafe 8 Resonancias e Influencias. En estas páginas explica Perlini que la primera tarea del pensamiento crítico es evitar las falsas esperanzas, por lo que asume sin ambigüedades que vivimos en un todo social reificado que no deja espacio para una vida auténtica. Ante esta situación caben salidas irracionales como la de S. Kierkegaard. La Escuela de Frankfurt y el teólogo danés reconocen la historia como mundo reificado, lo que empuja a este último a desanclar al sujeto respecto de un mundo carente de sentido y descubrir la esperanza en la interioridad absoluta como refugio interior. Para Kierkegaard, la separación entre sujeto y realidad es metafísica e inevitable, cuya conciliación no se alcanza en el plano horizontal de la inmanencia histórica sino en el recurso vertical a la trascendencia. Sin embargo, la Escuela de Frankfurt no acepta que el desgarramiento sea algo definitivo sino sólo un hecho histórico. La relación entre el sujeto y la realidad es dialéctica, lo que implica que, por un lado, su separación es histórica y epocal y nunca metafísica, y, por otro lado, su síntesis nunca es definitiva y siempre queda como algo proyectado y ansiado.

8 Cfr. Horkheimer, M., Adorno, Th. W., DI, p. 59-97/DA, p. 7-42. Consultar el capítulo Concepto de Ilustración.

9 Cfr. Horkheimer, M., Adorno, Th. W., DI, p. 79/DA, p. 26. 
- En el plano social, si la ciencia da por verdadero lo que existe de hecho, ésta nos pone frente a una realidad que no puede ser discutida críticamente sino sólo acatada. Por lo tanto, se excluye toda norma de conducta que no esté orientada a la adaptación a lo que hay.

La norma fundamental de conducta sería la autoconservación mediante la adaptación al orden social vigente, lo que convierte a la razón autónoma y a la conciencia libre en fuerzas disolventes del orden social. Así, las condiciones de trabajo en la sociedad industrial imponen un modelo de individuo genérico que resulta siempre clonado de otro. Este sí mismo, modelado como mercancía, es una consecuencia inevitable de la sociedad industrial, ya que esta, que exige autoconservarse mediante la pura adaptación mimética, genera un individuo conformista, el cual, frente a una igualdad represiva que anula la singularidad de cada uno, es siempre impotente. Horkheimer refiere que los verdaderos individuos nos son los inflados por la cultura de masas sino los mártires de la tiranía, ya que dichos mártires reflejan «la profunda resistencia humana contra la irracionalidad, una resistencia que siempre ha constituido el núcleo de la verdadera individualidad ${ }^{10}$. De este modo, el mantenimiento del orden social es imposible sin el autodominio del sí mismo como viviente. En la DI, el canto de las sirenas representa la promesa de una vida feliz placentera cuyo poder sobre nosotros es irresistible, y si cediéramos se disolvería la identidad humana y el lazo social. Sólo podemos no escucharla o bien como los galeotes, encerrados en su papel social sin oír ni entender otra cosa, o bien como Ulises que, asegurado al mástil de su identidad social, escucha el canto de las sirenas sólo como objeto de contemplación estética pero sin consecuencias en la praxis social aceptada ${ }^{11}$. Por lo tanto, el mantenimiento del lazo social exige que las formas de pensamiento y las categorías que constituyen la identidad del sujeto «no son, como enseña Durkheim, expresión de la solidaridad social, sino signo de la impenetrable unidad de sociedad y dominio» ${ }^{12}$.

En la actualidad, los que desde la impotencia sufren el dominio, por si fuera poco, carecen de conceptos teóricos que sirvan de cauce expresivo. La ciencia y su lenguaje, revestido de neutralidad, se quedan en una descomposición parcial del mundo en hechos concretos. Es cierto que ningún concepto puede aspirar nunca a lo Absoluto, pero si disolvemos todo concepto de lo Absoluto legitimamos el orden existente de facto. Por lo tanto, aún no pudiendo tomar ninguna de nuestras representaciones finitas por lo Absoluto, su negación es superadora ya que mantiene su momento de verdad. Nos confiesa Horkheimer, no sin cierta pesadumbre, que «la Ilustración ha devorado no sólo los símbolos, sino también a sus sucesores, los conceptos universales, y no ha dejado de la metafísica más que el miedo ante lo colectivo, del cual ella nació» ${ }^{13}$.

Es altamente llamativo cómo Horkheimer y Adorno se toman muy en serio el carácter trágico de nuestra experiencia histórica actual. "Por qué la humanidad, en lugar de entrar en un estado verdaderamente humano, se hunde en un nuevo género de barbarie » ${ }^{14}$, es la cuestión central que motiva la Dialéctica de la Ilustración. Partiendo como dato histórico de que «Los avances en el ámbito de los medios técnicos se ven acompañados

10 Cfr. Horkheimer, M., CrI, p. 168/KiV, p. 152.

11 Cfr. Horkheimer. M., Adorno, Th. W., DI, p. 97-129/DA, pp. 42-74. Consultar el apartado Excursus I: Odiseo, o mito e Ilustración.

12 Cfr. Horkheimer, M., Adorno, Th. W., DI, p. 75/DA, p. 23.

13 Cfr. Horkheimer, M., Adorno, Th. W., DI, p. 77/DA, p. 24.

14 Cfr. Horkheimer, M., Adorno, Th. W., DI, p. 51/DA, p. 1. 
por un proceso de deshumanización ${ }^{15}$ la causa para Horkheimer de tal situación es meridiana; la razón es esencial y originariamente instrumento de dominio. Desde este punto de vista, en primer lugar, no se reconoce resistencia o alteridad en la naturaleza sino que su esencia queda reducida a su lado cuantificable. Por lo tanto, nuestro saber acerca de ella no está orientado a la verdad sino a desarrollar operaciones más eficaces que mejoren nuestro dominio. En segundo lugar, al olvidarse u ocultarse la base natural del sujeto, se bloquea una discusión racional acerca de los verdaderos fines del hombre, por lo que la «liquidación operada por ella de todo lo que es en sí mismo vinculante permite al dominio decretar de forma soberana y manipular las obligaciones que en cada caso le convienen ${ }^{16}$, o de otra manera, que la razón misma queda como mero instrumento al servicio del modo de producción dominante. En tercer lugar, los hechos y el éxito como criterio de verdad de la razón dan lugar a un modelo social en el que no cabe un distanciamiento crítico respecto de la realidad vigente sino que, al contrario, la unidad del colectivo, lejos de constituirse en una verdadera intersubjetividad, genera igualdad represiva que niega la singularidad de cada uno. La adaptación a lo que hay como norma fundamental atomiza la sociedad y hace que el individuo se vuelva conformista e impotente ${ }^{17}$. Por este motivo, la intersubjetividad como «la posibilidad de un acuerdo sin coerción y de reconocimiento sin violencia $»^{18}$ es, desde un punto de vista histórico, el tema de nuestro tiempo y no el supuesto de la reflexión filosófica.

Sin embargo, para Horkheimer y Adorno en la DI, estas tres tesis están en conexión necesaria e inevitable, lo que da lugar a que la finalidad última de dicha obra no sea revocar en la razón su tendencia instrumentalista. La autodisolución del pensamiento como instrumento de dominio es a estas alturas de la historia una regresión imposible de invertir, ya que «si la razón subjetiva disolvió la base filosófica de las convicciones fideístas que habían sido parte esencial de la cultura occidental, ello fue posible porque esta base se había revelado como demasiado débil....Su revitalización es, en consecuencia, de todo punto artificial: cumplen el objetivo de rellenar un vacío ${ }^{19}$. En RyA ${ }^{20}$, nos explica Horkheimer que la desintegración de la razón, como concepto fundamental de la civilización occidental, se inicia cuando la crítica, al incluirse en el concepto de razón, termina anulando el concepto de sí misma. Aplicando los criterios de infalibilidad, severidad, claridad y precisión, la misma razón se vuelve impotente para apuntar a una realidad más allá de lo existente.

Por ello, la finalidad de la DI es que en base al precio pagado tomemos conciencia de la profunda ambigüedad que acompaña al pensamiento en constante progreso. El progreso, como movimiento universal del espíritu, se ha producido a costa de extender las relaciones de dominio, del aislamiento y empequeñecimiento del hombre. Con gran oportunidad se cita en este punto a Nietzsche:

«Llevar la Ilustración hasta el pueblo, de modo que los sacerdotes se hagan sacerdotes con mala conciencia; y lo mismo hay que hacer con el estado. Tarea de la Ilustración es mostrar la entera conducta de los príncipes y gobernantes como mentira intencionada [...] El engaño al que se ve sometida la multitud en este punto, por ejemplo, en toda

15 Cfr. Horkheimer, M., CrI, p. 43/KiV, p. 13.

16 Cfr. Horkheimer, M., Adorno, Th. W., DI, p. 140/DA, p. 84.

17 Cfr. Horkheimer, M., Adorno, Th. W., DI, p. 165-213/DA, pp. 108-151. Consultar el apartado La industria cultural. La Ilustración como engaño de masas.

18 Cfr. Habermas, J., CI, p. 183/ EuI, p. 222.

19 Cfr. Horkheimer, M., CrI, p. 92/KiV, p. 66.

20 Cfr. Horkheimer, M., Teoría Crítica, p. 139-176/VuS, pp. 7-57. Consultar el artículo Razón y Autoconservación. 
democracia es precioso: ¡el empequeñecimiento y la gobernabilidad de los hombres son perseguidos como progreso! $\aleph^{21}$

El mismo carácter aporético de la DI refleja la imposibilidad de la autosuperación de la razón como instrumento de dominio. Sin embargo, el terreno en el que sí se mueve legítimamente la DI es en el de la denuncia del principio ciego del dominio como falso absoluto. Para Horkheimer, un principio es un falso absoluto cuando su aplicación involucra una denegación u olvido del sufrimiento de las víctimas, y la razón instrumental, al reducir la Naturaleza a materia prima sin otro fin que el dominio mismo, oculta «una historia de sufrimiento infinito» ${ }^{22}$ en ella. Por este motivo, en la $\mathrm{CrI}$ se dice que la función de la filosofía es llevar a lenguaje tanto los anhelos de los oprimidos como la violencia sobre la naturaleza, aunque sólo con la finalidad de que el falso absoluto se reconozca a sí mismo como tal. En efecto, la naturaleza no reconciliada o sometida es la viva imagen del dominio, y sólo «en la humildad en la que el espíritu se reconoce como dominio y se revoca en la naturaleza se disuelve su pretensión de dominio ${ }^{23}$. El pensamiento se opone al dominio cuando se reconoce a sí mismo en la imagen de la naturaleza mutilada o sometida. Cuando lo hace, es decir, cuando autorreflexiona elevando a concepto su propia praxis, entonces se mitiga el dominio. Sólo así, mediante «el recuerdo de la naturaleza en el sujeto, en cuya realización se encierra la verdad desconocida de toda cultura, la Ilustración se opone al dominio en cuanto tal ${ }^{24}$.

Respecto de la DI, la CrI pretende ir algo más allá del momento crítico o destructivo de la razón. En esta obra Horkheimer no acepta el escepticismo total, ya que este puede ser tan conservador como la vuelta a la vieja metafísica, que pretendía que el pensamiento cesara para crear una esfera especial de verdades. La vuelta en la actualidad a la metafísica de lo absoluto es la prueba visible de cuán poco convincentes y dudosas son dichas supuestas verdades, cuya finalidad específica es mucho más servir a los grupos de poder dominantes que criticar la realidad ${ }^{25}$. En este sentido dice Horkheimer:

Hoy pervive la tendencia a establecer un principio absoluto como poder real o un poder real como principio absoluto; se diría que el valor supremo sólo puede ser visto como verdaderamente absoluto cuando es a la vez el poder supremo ${ }^{26}$.

En esta obra mantiene que la razón es esencial y originariamente instrumento de dominio, y si ésta tiene como intención propia descubrir la verdad no es porque pueda acceder a una objetividad absoluta ya que «los conceptos dispuestos y graduados de acuerdo con el orden de su generalidad reflejan más la represión de la naturaleza por los hombres que la estructura de la naturaleza misma ${ }^{27}$. Nuestros conceptos no se corresponden con la cadena eterna del ser ya que, en su origen histórico, lo que nos llevó a formarlos no fue captar el orden lógico de la realidad sino el móvil del poder, primero, para mantener las relaciones de dominio en el seno de la ciudad estado, y luego, extrapolándolas a la naturaleza, sometiéndola. Por lo tanto, la armonía entre hombre y naturaleza se reduce al falso supuesto de que el universo esté hecho a imagen y semejanza

21 Nietzsche, F., Nachlass, en Werke, Grossoktavansgabe, Leipzig 1904, vol. XIV, p. 206; vol. XV, p. 235, citado en: Horkheimer, M., Adorno, Th. W., DI, p. 98/DA, pp. 42-43

22 Cfr. Horkheimer, M., CrI, p. 141/KiV, p. 122.

23 Cfr. Horkheimer, M., Adorno, Th. W., DI, p. 92/DA, p. 39.

24 Cfr. Horkheimer, M., Adorno, Th. W., DI, p. 93/DA, p. 39.

25 Cfr. Horkheimer, M., CrI, p. 89-115/KiV, pp. 63-93. Consultar el capítulo Panaceas universales antagónicas.

26 Cfr. Horkheimer, M., CrI, p. 97/KiV, pp. 72-73.

27 Cfr. Horkheimer, M., CrI, p. 183/KiV, p. 169. 
del hombre. Por este motivo, «la ontología filosófica es inevitablemente ideológica, porque trata de oscurecer y velar la separación entre hombre y naturaleza desmentida por doquier por los gritos de los miserables y de los desheredados ${ }^{28}$.

La orientación a la verdad de la razón, tanto en la CrI como en la DI, reside en reflexionar sobre su tendencia al dominio como su condición natural. En la medida que se abre a la búsqueda de la verdad acerca de sí misma ya no rige sólo en ella la lógica inconsciente del dominio. Al descubrir el plano de la dimensión autocrítica o autorreflexiva de la razón, ésta ya no es sólo instrumento de dominio sino que puede ser «un instrumento de reconciliación ${ }^{29}$ con la naturaleza. En efecto, decimos, a lo sumo, que los procesos de toma de conciencia pueden cambiar el rumbo de los acontecimientos ya que, para Horkheimer, el conocimiento de una contradicción no necesariamente nos lleva a su superación.

¿Dónde encuentra la filosofía la llave de esta posibilidad de transformación?

La filosofía no es una herramienta ni una receta; sólo puede bosquejar por anticipado el curso del progreso tal y como este viene determinado por necesidades lógicas y reales; al hacerlo puede anticipar la reacción de horror y resistencia que provocará la marcha triunfal del hombre moderno ${ }^{30}$.

La diferencia con respecto al planteamiento de la DI reside en que el punto de referencia para que la razón se reconozca a sí misma como instrumento de dominio no es la naturaleza como "historia de sufrimiento infinito " ${ }^{31}$, sino el contenido de verdad de todo contenido histórico concreto. La filosofía sondea las ideas regulativas que están en el trasfondo social de cada momento histórico, y habrá verdad en la historia en la medida en que dichos principios conceptuales sean vigentes y efectivos. Por lo tanto, el carácter positivo o superador de la razón reside en que «las ideas culturales fundamentales conllevan un contenido de verdad, y la filosofía debería medirlas en relación con el trasfondo social del que proceden. La filosofía combate el hiato entre ellas y la realidad. Confronta lo existente en su nexo histórico de interrelaciones con la pretensión de sus principios conceptuales con vistas a criticar la relación entre ambos e ir así más allá de ellos ${ }^{32}$. Que nuestras ideas culturales tienen contenido de verdad no significa que posean una pretensión absoluta de verdad.

La filosofía toma en serio los valores existentes, pero insiste en que se convierten en elementos de un todo teórico que revela su relatividad ${ }^{33}$.

La filosofía niega tanto la pretensión de verdad absoluta de la ideología dominante como la de la realidad fáctica, y sobre la ruina de ambos falsos absolutos intenta salvar las verdades relativas ${ }^{34}$.

\subsection{La praxis comunicativa entre sujetos como tema de nuestro tiempo en Horkheimer.}

¿Dónde está el punto en común a partir del cual extraen consecuencias distintas Horkheimer y Habermas en sus respectivos planteamientos? Para ambos la razón es

28 Cfr. Horkheimer, M., CrI, p. 183/KiV, p. 169.

29 Cfr. Horkheimer, M., CrI, p. 180/KiV, p. 165.

30 Cfr. Horkheimer, M., CrI, p. 171/KiV, p. 155.

31 Cfr. Horkheimer, M., CrI, p. 141/KiV, p. 122.

32 Cfr. Horkheimer, M., CrI, p. 184/KiV, p. 170.

33 Cfr. Horkheimer, M., CrI, p. 184/KiV, p. 170 filosofía.

Cfr. Horkheimer, M., CrI, p. 169-187/KiV, pp. 153-175. Consultar el apartado Sobre el concepto de 
instrumento de dominio, pero no para los dos lo es de forma esencial y originaria. Ya hemos expuesto cómo para Horkheimer la razón no es instrumento de dominio epocal e históricamente sino que deviene totalitaria por su esencia y fundamento. En la CrI nos dice literalmente que «la autodisolución de la razón como sustancia espiritual obedece a una necesidad interna» ${ }^{35}$, y en el mismo prólogo citado de 1967 refiriéndose a Nietzsche dice que éste denunció la creencia en la razón autónoma como un síntoma de atraso. Todas las filosofías que apuntan a la intuición de esencias ya sea en el plano lógico como Husserl o, así como en Scheler, en el plano de las estructuras morales, caen todas ellas desde sus comienzos bajo «signo de lo restaurativo» ${ }^{36}$. Para Horkheimer «la teoría debe reflejar y dar curso expresivo hoy al proceso, a la tendencia socialmente condicionada, al neopositivismo, a la instrumentalización del pensamiento, así como a los vanos intentos de salvación» ${ }^{37}$. Con esto último, se refiere a los anteriormente citados proyectos de recuperación de la razón concebida como la capacidad para concebir ideas eternas que nos sirvan como fines. A este respecto, la función de la teoría crítica es despejar tales proyectos como intentos, imposibles tanto desde un punto de vista histórico como metafísico, de restablecer una esfera de verdades absolutas, las cuales sirven más como instrumento al servicio de los poderes fácticos que como referente crítico de la realidad social. Sin embargo, en RyA Horkheimer reconoce que los objetivos humanos no se hallan inmediatamente dados por naturaleza sino por la razón como categoría social, y que estos, aunque impliquen abjurar de la propia razón subjetiva, son imprescindibles para el mantenimiento de la cooperación humana. Por ello, son irrenunciables pero a la vez imposibles de enunciar, dado el abismo lógico entre el conocimiento racional o científico y la esfera de la pluralidad de objetivos, que dependen de la arbitrariedad subjetiva. En definitiva, aunque la universalidad de la razón significa conformidad con los intereses de todos los individuos, en la sociedad de clases con intereses irreconciliables, «la apelación teorética a la universalidad de la razón posee siempre rasgos característicos de falsedad y represión ${ }^{38}$.

De la razón como instrumento de dominio, por sí misma y por esencia, deviene por necesidad interna un determinado modelo de praxis comunicativa entre sujetos. En Horkheimer la comunicación entre sujetos no es cooriginaria con la tendencia al dominio de la naturaleza sino derivada de ella. Si nos relacionamos con la naturaleza reduciéndola a materia prima, entonces se generan en la sociedad unas condiciones de trabajo orientadas hacia la mejora de la eficacia y de la productividad. El trabajo, en tanto que actividad humana, impregna y transforma todas las relaciones sociales hasta el punto que, en palabras de Horkheimer, «la autoconciencia de los hombres ha pasado a identificarse con su función en el sistema dominante ${ }^{39}$. El individuo reprime los anhelos no adecuados al modelo existente, lo que genera en él tal resentimiento que si se disolviera la coacción se volvería contra todo el orden social ${ }^{40}$. Por esta causa la autodenegación del individuo no va más allá de la sociedad industrial, éste es en el fondo un medio al servicio de la mejora de la producción ya que «el yo es percibido como algo que está vinculado a funciones de dominio, mando y organización.... En ningún momento ha podido el yo borrar de sí la mácula de su origen en el sistema de dominio

35 Cfr. Horkheimer, M., CrI, p. 40/KiV, p. 8.

36 Cfr. Horkheimer, M., CrI, p. 40/KiV, p. 8.

37 Cfr. Horkheimer, M., CrI, p. 40/KiV, p. 8.

38 Cfr. Horkheimer, M., RyA, p. 148/VuS, p. 17.

39 Cfr. Horkheimer, M., CrI, p. 155/KiV, p. 137.

40 Cfr. Horkheimer, M., CrI, p. 143-169/ KiV, pp. 124-153. Consultar el apartado Ascenso y decadencia del Individuo. 
social $\aleph^{41}$. En RyA, cuestionándose si se puede justificar racionalmente el sacrificio de los instintos del individuo al poder del Estado, concluye que la racionalidad del sacrificio depende del status social, es decir, está en función de la propiedad. Sólo la conservación de la propiedad funda racionalmente la sumisión al Estado, pero, desde la miseria, no se puede justificar racionalmente la renuncia a los instintos. La masa social debe renunciar a sí misma, sin lo cual es imposible el progreso en la sociedad industrial, pero tampoco la razón puede producir por sus propias fuerzas la justificación que el hombre moderno necesita. Por eso, la educadora de la masa fue primero la Reforma religiosa, que, mediante la introyección del martirio en el alma humana, propaga un individuo frío y racional, y después, bajo el predominio de los monopolios, el sistema cultural, tendente a anular la oposición del individuo respecto del mundo objetivo mediante la imposición de esquemas dominantes que operan en la conciencia. Si cualquier cosa vivida por los individuos es una función del sistema convencional de conceptos de la sociedad, entonces la individualidad está plenamente neutralizada y la oposición crítica ya no es posible.

También en Horkheimer cabe invertir la relación entre causa y efecto, a saber, que el sistema de dominio social plasmado en las formas de división del trabajo deriva en una concepción del sujeto reducido a su función dominadora y clasificadora. Por lo tanto, si no existe una praxis comunicativa verdaderamente intersubjetiva entre sujetos, se debe a que las condiciones de trabajo en la sociedad generan un modelo de individuo en el que sus «formas de pensamiento no son, como enseña Durkheim, expresión de la solidaridad social, sino signo de la impenetrable unidad de sociedad y dominio» ${ }^{42}$. Dado que el sujeto replica en sí las categorías intelectuales que favorecen la reducción de la naturaleza a materia prima sin otro fin que el dominio mismo, dado que el ser humano bajo este esquema se convierte en un ser genérico y duplicado de otro, entonces éste es sólo capaz de experimentar lo que previamente ha sido ya experimentado. Acordándose del esquematismo kantiano nos dice Horkheimer:

La verdadera naturaleza del esquematismo, que hace concordar desde fuera lo universal con lo particular, el concepto y el caso singular, se revela finalmente en la ciencia actual como el interés de la sociedad industrial ${ }^{43}$.

$\mathrm{El}$ aparato conceptual de la ciencia y la misma industria cultural hace hoy las veces del esquematismo trascendental de Kant. Éste, al referir la multiplicidad sensible de los datos inmediatos a conceptos fundamentales, no permite percibir ni experimentar nada que no haya sido ya anticipado por el esquematismo de la producción, es decir, prefigurado con arreglo a los intereses de la sociedad industrial. Liquidándose la subjetividad y la conciencia libre, reducido el individuo a su función en el sistema y sin otras categorías intelectuales que las de la razón instrumental, sólo entiende del dominio del otro y del autodominio de sí. Precisamente por eso «la autodenegación del individuo en la sociedad industrial no tiene objetivo alguno que pudiera ir más allá de la propia sociedad industrial. Tal renuncia genera racionalidad en lo que hace a los medios e irracionalidad en lo que hace a la existencia humana» ${ }^{44}$.

El dominio, sin otra finalidad que el dominio mismo, implica que el sujeto vive irracionalmente en lo que toca a los fines últimos de la existencia humana. El dominio se enfrenta al individuo singular como lo universal, como una racionalidad inmanente

41 Cfr. Horkheimer, M., CrI, p. 125-126/KiV, pp. 104-105.

42 Cfr. Horkheimer, M., Adorno, Th. W., DI, p. 75/DA, p. 23.

43 Cfr. Horkheimer, M., Adorno, Th. W., DI, p. 131/DA, p. 76.

44 Cfr. Horkheimer, M., CrI, p. 116/KiV, p. 94. 
a la realidad y él mismo, con su actividad, contribuye a actualizarlo y mantenerlo, ya que rechaza, como si fueran fuerzas aniquilantes o destructoras del orden social, todo aquello que no encaje como puro medio en el contexto instrumental de la acción cuyo fin último sería la autoconservación en el sistema.

Puesto que el dominio es una consecuencia lógica inevitable de la sociedad industrial, o al contrario, como la sociedad industrial es consecuencia lógica del dominio, no puede existir una auténtica comunicación libre entre sujetos. Desde el supuesto de una concepción de la naturaleza como objeto de dominación total, la regresión de las masas acontece por necesidad lógica y consiste en «la incapacidad de poder oír con los propios oídos aquello que no ha sido aún oído, de tocar con las propias manos aquello que no ha sido aún tocado» ${ }^{45}$. De esta manera, «los hombres son reducidos....a simples seres genéricos, iguales entre sí por aislamiento en la colectividad coactivamente dirigida» ${ }^{46}$. Las condiciones de trabajo han impuesto en su lugar una masa social conformista e impotente frente al aparato técnico, no epocal e históricamente sino por la necesidad interna de su desarrollo:

La impotencia de los trabajadores no es sólo una artimaña de los patrones, sino la consecuencia lógica de la sociedad industrial, en la que se ha transformado finalmente el antiguo destino bajo el esfuerzo por sustraerse a él ${ }^{47}$.

Sobre la base de una relación con la naturaleza como objeto de dominación total, la praxis intersubjetiva es incapaz de generar fines últimos para la actividad humana. En efecto, mientras que el pensamiento caiga bajo la lógica inconsciente y ciega del dominio la naturaleza seguirá siendo sólo materia prima, pero si la naturaleza no reconciliada o sometida se contemplara como la viva imagen del dominio, entonces el pensamiento podrá iniciar un nuevo camino de reflexión en el que éste se reconozca a sí mismo como instrumento de dominio. Por eso, aunque el conjunto social haya resultado ciego y sin salida por inevitable necesidad, no por ello deja de ser un hecho problemático. La Ilustración se opone al dominio en cuanto tal «mediante este recuerdo de la naturaleza en el sujeto, en cuya realización se encierra la verdad desconocida de toda cultura» ${ }^{48}$. Este recuerdo de la naturaleza en el sujeto como «historia de sufrimiento infinito» lleva a la razón a ser algo más que tendencia al dominio. Dicho recuerdo la abre a la dimensión autocrítica y autorreflexiva acerca de sí misma y con ello descubre la verdad, y no el dominio del otro, como su intencionalidad más propia. Y en el camino hacia la verdad, la razón es más que un instrumento para dominar, es un instrumento de reconciliación:

La razón sólo puede ser algo más que naturaleza en la medida en que se hace concretamente consciente de su "condición natural» —que consisten en su tendencia al dominio-, la misma tendencia que paradójicamente la enajena de la naturaleza. De este modo, en la medida en que es un instrumento de reconciliación, pasa a ser, a la vez, algo más que un instrumento. Los cambios de rumbo, los progresos y los retrocesos en este empeño reflejan la evolución de la definición de filosofía ${ }^{49}$.

No hay aquí una crítica regresiva o conservadora a la Ilustración que pretenda un retorno romántico a la Naturaleza. Ni hay salidas irracionales ya que «la libertad de la sociedad es inseparable del pensamiento ilustrado $»^{50}$, ni tampoco con la razón, dada la

45 Cfr. Horkheimer, M., Adorno, Th. W., DI, p. 89/DA, p. 36.

46 Cfr. Horkheimer, M., Adorno, Th. W., DI, p. 89/DA, p. 36.

47 Cfr. Horkheimer, M., Adorno, Th. W., DI, p. 89/DA, p. 36.

48 Cfr. Horkheimer, M., Adorno, Th. W., DI, p. 93/DA, p. 39.

49 Cfr. Horkheimer, M., CrI, p. 180/KiV, p. 165.

50 Cfr. Horkheimer, M., Adorno, Th. W., DI, p. 53/DA, p. 3. 
ecuación originaria entre ésta y la tendencia el dominio, podemos superar el conflicto con la Naturaleza. Sólo el recuerdo de lo olvidado, es decir, de la sintonía con la Naturaleza le puede ayudar a oponerse a su tendencia al dominio y convertir la razón en instrumento de reconciliación.

\section{LA UNIDAD EPOCAL E HISTÓRICA ENTRE RAZÓN Y DOMINIO EN HABERMAS}

\subsection{Los distintos intereses del conocimiento y su génesis en Conocimiento e Interés}

En nuestra investigación se ha comparado al primer Habermas, es decir, el correspondiente al periodo de la lucha contra el cientificismo entre los años 1961 y 1968, con el Horkheimer de la segunda época, el que va desde el 1942 a 1950. Se ha preferido la primera época del pensamiento de Habermas porque es el momento en el que discute con la primera generación de Escuela de Frankfurt en sus mismos términos. Si toda la Escuela de Frankfurt comienza con una concepción de la razón contextual e interesada, es prioritario cuestionarse si la razón está constituida esencial y originariamente por el interés de dominio o si más bien, por el contrario, hay en ella vigentes diversos intereses y, en consecuencia, cuál de ellos debería ser principio rector del conocimiento. Esta es la tarea que Habermas acomete en la primera época de su pensamiento: demostrar que la reducción de la razón al interés de dominio es epocal e histórica, no esencial y originaria, por lo que existen otros intereses vigentes y efectivos en las diferentes ciencias. Pretende aquí una crítica interna a la primera generación de frankfurtianos, más que, como en épocas posteriores de su pensamiento, y desde el paradigma ajeno a Horkheimer de la teoría de la comunicación, interesarse por los fundamentos normativos de la crítica ${ }^{51}$.

La convergencia entre Horkheimer y el planteamiento habermasiano de CI arranca, como se explicitó más arriba, de que para ambos la razón es instrumento de dominio pero a su vez divergen en que no para los dos la razón lo es de forma esencial y originaria.

Todo el planteamiento de Habermas de CI comienza en la exposición como principio de la conexión entre conocimiento e interés. En efecto, intereses cognoscitivos diferentes implican distintos formas de conocer la realidad y, a la inversa, diferentes modos de conocer la realidad suponen intereses cognoscitivos distintos. Desde dicho principio, dado que de facto existen modos de conocer la realidad distintas, se puede demostrar la existencia de intereses del conocimiento independientes entre sí. Y si existen intereses cognitivos distintos, entonces no se puede reducir la razón de forma exclusiva y excluyente a uno de los intereses de la razón, es decir, a instrumento de dominio, sino que, parejamente con dicha tendencia, la razón también puede ser comunicativa o práctica. Así pues, y este es el término de llegada del planteamiento habermasiano, la existencia

51 Cfr. Moritz, P., Kritik des Paradigmenwechsels. Mit Horkheimer gegen Habermas, Lüneburg, Dietrich zu Klampen Verlag, 1992. Consultar, pp. 184-199, el epígrafe II.3 Funktion der Habermasschen Kritk am Mimesis Begriff für die methodische Konstruktion einer Theorie der Gesellschaft. En este capítulo, Moritz objeta a Habermas que mucho más prioritario que asegurar la validez normativa de la crítica, sería realzar, en el ejercicio mismo de la crítica, las contradicciones de nuestra época. Este camino deja al ejercicio autocrítico de la razón en equilibrio inestable, con la sola referencia, como canon crítico normativo, de categorías meramente aludidas, pero nunca expresadas positivamente. Pero es preferible una razón crítica que, aún permaneciendo en constante movimiento y sin categorías normativas estables, sea capaz de captar las contradicciones de una época en toda su crudeza, que otra racionalidad que, salvando la consistencia normativa de la crítica, disimule los antagonismos sociales. 
de intereses de conocimiento diversificados desactiva de raíz la crítica total a la razón como instrumento de dominio.

En la primera parte de la obra ${ }^{52}$ desarrolla la crítica de Hegel a Kant y la crítica de Marx a Hegel. Con Hegel, el sujeto no es autoposición inmediata de sí sino que lo primero para la conciencia es el mundo cotidiano o natural al que pertenecemos y en el que nos encontramos. Por lo tanto, si la conciencia se quiere saber a sí misma, y por conocerse a sí misma también constituirse, ha de recorrer un proceso de formación partiendo de lo más inmediato, la certeza sensible. De este modo, Hegel pone sobre la mesa que sujeto y mundo no son realidades independientes sino en mutua interrelación, y que interactuando entre sí recíprocamente se constituyen. Así sujeto y mundo, en su mutua interrelación, están sometidos a un proceso recíproco de formación en el que ambos resultan constituyentes y constituidos:

La experiencia fenomenológica, contrariamente a lo que sucede con la empírica, no transcurre dentro de los límites de esquemas establecidos mediante una fundamentación trascendental; en la construcción de la conciencia que se nos aparece intervienen más bien, por el contrario, las experiencias fundamentales en las que las transformaciones de tales esquemas de la comprensión del mundo y del hacer se han decantado ${ }^{53}$.

Sin embargo, en el movimiento genético constructivo que supera la falsa conciencia de sí y del mundo, no hay involucrado ningún interés del sujeto por saberse a sí mismo, sino que cada figura de la conciencia surge como consecuencia lógica de la anterior. Si todo el camino fenomenológico acontece por necesidad interna, esto introduce en todo el proceso una profunda ambigüedad:

La perspectiva propia del conocimiento absoluto es la de avanzar mediante una necesidad inmanente desde la experiencia fenomenológica, pero precisamente en cuanto que este saber es absoluto no necesita propiamente de la justificación que podría proporcionarle la autorreflexión fenomenológica ${ }^{54}$.

Por un lado, Hegel radicaliza la crítica del conocimiento y por ello no parte de supuestos previos, sólo de la duda radical o conciencia crítica que se legitima a sí misma. Por consiguiente, el punto de vista del saber puro o ciencia estricta como final del camino fenomenológico, es un saber siempre problemático y se produce como consecuencia de la crítica del conocimiento, es decir, tiene como condición previa el interés del sujeto por liberarse de la falsa conciencia. Pero, por otro lado, «la experiencia fenomenológica se mantiene y se ha mantenido siempre en el medio de un movimiento absoluto del espíritu, y por ello debe desembocar necesariamente en el saber absoluto ${ }^{55}$. En efecto, si el proceso acontece por necesidad inmanente, entonces el punto de vista del saber puro no es resultado problemático sino el presupuesto previo no sometido a crítica de toda la cadena de figuras de la conciencia. Por ello la crítica del conocimiento no es total sino que "asume como dado un conocimiento de lo absoluto» ${ }^{56}$, el cual guía las modificaciones de la relación trascendental de sujeto y objeto hasta terminar por automanifestarse.

La crítica del conocimiento en Hegel queda ambiguamente inconclusa ya que los principios rectores del conocimiento son, por una parte, el interés del sujeto por

52 Cfr. Habermas, J., CI, p. 11-75/EuI, pp. 11-88. Consultar el apartado La crisis de la crítica del conocimiento.

53 Cfr. Habermas, J., CI, p. 28/ EuI, p. 31.

54 Cfr. Habermas, J., CI, p. 17/ EuI, p. 18.

55 Cfr. Habermas, J., CI, p. 28/ EuI, p. 30.

56 Cfr. Habermas, J., CI, p. 17/ EuI, p. 18. 
liberarse de la falsa conciencia, y por otra parte, el Absoluto que se da y se muestra en la conciencia como motor de la experiencia fenomenológica.

Marx, al considerar al hombre como un "ente objetivo» ${ }^{57}$, decanta la ambigüedad en Hegel sobre el principio rector que guía la crítica del conocimiento hacia el lado de los intereses del sujeto. Según dicha consideración, la "parte activa ${ }^{58}$ del hombre es su condición material o sensible, lo que implica que el sujeto de dicha actividad no es el espíritu, sino la especie humana concreta que reproduce su vida bajo condiciones naturales. La actividad, por la cual el sujeto pone las condiciones para la reproducción de la vida, es el trabajo. Por eso, el trabajo es un proceso o poder natural de la especie humana para su propia reproducción a la vez que una actividad trascendental, ya que él mismo y la naturaleza circundante son resultado de dicha actividad como praxis transformativa.

Sin embargo, Marx reduce los intereses que guían la autoconstitución de la especie humana a la acción técnico-instrumental sobre la naturaleza, es decir, al desarrollo de las fuerzas productivas cuyo valor límite es la emancipación de la naturaleza externa y la consiguiente organización social como un autómata. Pero a este último valor límite no se llega sólo con el desarrollo de la producción, hay que apropiarse de las fuerzas esenciales de trabajo exteriorizadas. De este modo, el proceso de autoconstitución de la especie humana consiste en "producción y apropiación, exteriorización y apropiación de la fuerza esencial exteriorizada ${ }^{59}$ y Marx mismo concibe la reflexión según el modelo de la producción. Al partir tácitamente de esta premisa es consecuente que no distinga entre el status lógico de las ciencias de la naturaleza y las de la crítica.

Al dar prioridad a la producción y al trabajo para la constitución de la especie humana, entonces el sujeto social se constituye unilinealmente como resultado de la acción técnico-instrumental sobre la naturaleza. Si la acción técnico-instrumental orientada al dominio de la naturaleza y al desarrollo de las fuerzas productivas se basa en el conocimiento de las leyes de la naturaleza, así mismo también, en el plano social, el control de los procesos sociales depende del conocimiento de las leyes de la sociedad, las cuales no sólo son independientes de la conciencia libre sino que además la determinan. Por lo tanto, la finalidad de las ciencias naturales y de las ciencias sociales es la misma; el control de los procesos sociales.

Marx termina suprimiendo una teoría del conocimiento como crítica en favor de una ciencia universal de las leyes de la sociedad de cuyo conocimiento depende el control de los procesos sociales. Es imposible una autorreflexión crítica del sujeto que no sea una mera reconstrucción de la historia de la especie, ya que la interacción comunicativa entre sujetos y la misma conciencia subjetiva es resultado del desarrollo de la producción y del trabajo.

En conclusión, Marx acierta en que la autoconstitución de la especie humana está guiada por intereses o principios rectores del conocimiento, pero yerra en que reduce éstos, de forma exclusiva y excluyente, a la apropiación de la naturaleza por el trabajo social. La interacción comunicativa entre sujetos no genera los fines del dominio de la naturaleza sino que ocurre más bien al contrario; es el potencial creciente de disposición técnica sobre la naturaleza el que genera la posibilidad material para nuevas formas de organización política e institucional.

\footnotetext{
57 Cfr. Habermas, J., CI, p. 34/ EuI, p. 38.

58 Cfr. Habermas, J., CI, p. 34/ EuI, p. 38.

59 Cfr. Habermas, J., CI, p. 53/ EuI, p. 61.
} 
Mientras que en Marx las relaciones de producción y la misma intersubjetividad son resultado del grado de desarrollo de las fuerzas de producción, en Habermas «la destrucción relativa de la relación ética se mide sólo por la diferencia entre el grado efectivo de represión exigida institucionalmente y el grado de represión necesaria en cada estadio de las fuerzas productivas. Esta diferencia es la medida de la dominación que es objetivamente superflua» ${ }^{60}$.

\subsection{La cooriginariedad en Habermas de los distintos intereses del conocimiento.}

Habermas, en la primera parte del libro, ha probado la conexión entre el conocimiento y el interés, aunque, de momento, sólo tenemos la acción técnico-instrumental sobre la naturaleza como único interés. Como esta tesis nos llevaría sin remedio a una crítica total a la razón como instrumento de dominio, Habermas necesita demostrar la existencia de otros intereses de la razón.

Esto último, es precisamente la tarea de la segunda parte de la obra ${ }^{61}$. Su método de prueba es demostrar la diferencia entre las ciencias naturales y las ciencias críticohermenéuticas, y así, de esta manera, dado que de facto existen modos de conocer la realidad distintos, se puede demostrar la existencia de intereses del conocimiento independientes entre sí. Y si existen intereses cognitivos distintos, entonces no se puede reducir la razón de forma exclusiva y excluyente a uno de los intereses de la razón, es decir, a instrumento de dominio sino que parejamente con dicha tendencia la razón también puede ser comunicativa o práctica.

Habermas acomete la diferenciación entre ciencia natural y ciencia hermenéutica mediante una crítica a la metodología positivista. La lógica de la ciencia positivista mantiene que las reglas para la comprobación y constitución de las teorías son capaces de garantizar que éstas se correspondan con la realidad. De este modo, el positivismo procede como si las reglas sintéticas para la constitución de teorías científicas capaces de describir el mundo no tuvieran su origen en el sujeto, como si la metodología científica garantizara que fueran perfectamente isomórficas con la realidad y, al contrario, éstas no tuvieran su origen en el sujeto que conoce. Pero si el conocimiento es meramente descriptor de la realidad, entonces no hay espacio para las ciencias hermenéuticas, ya que estas, frente a las ciencias empíricas que vierten la experiencia posible en las expresiones universales de los lenguajes teóricos, pretenden «esclarecer la estructura del lenguaje ordinario en el aspecto en el que permite precisamente lo que la sintaxis del lenguaje puro prohíbe: hacer comunicable, aunque indirectamente, lo individual inexpresable» ${ }^{62}$. La hermenéutica no es necesaria en los lenguajes puros en los que la acción comunicativa no afecta al contenido del juicio, pero sí se requiere cuando los interlocutores protagonistas de una acción comunicativa afectan al contenido del juicio, es decir, cuando el mensaje recibe en la interlocución un sentido individual. Por lo tanto, en el plano de la praxis vital comunicativa, la hermenéutica busca comprender las manifestaciones individuales de la vida que no logran recoger adecuadamente las categorías generales del lenguaje. Con ello, se pretende asegurar «la intersubjetividad de la comprensión en la comunicación lingüística ordinaria en la acción bajo normas comunes ${ }^{63}$. La intersubjetividad de la comprensión se refiere a «una comprensión recíproca entre

60 Cfr. Habermas, J., CI, p. 69/ EuI, p. 80.

61 Cfr. Habermas, J., CI, p. 119-147/EuI, pp. 143-178, el apartado Autorreflexión de las ciencias de la naturaleza y, CI., p. 168-193/EuI, p. 204-234, el apartado Autorreflexión de las ciencias del espíritu.

62 Cfr. Habermas, J., CI, p. 170-171/ EuI, p. 206.

63 Cfr. Habermas, J., CI, p. 182/ EuI, p. 221. 
individuos... que hace posible la forma de un consenso sin coerciones y el tipo de una intersubjetividad discontinua, de los que depende la acción comunicativa» ${ }^{64}$. Por este motivo, si la comprensión recíproca entre individuos excluyera las manifestaciones individuales de la vida, entonces «la intersubjetividad de la comprensión se hace rígida o se derrumba, y queda destruida una condición de supervivencia, que es tan elemental como la condición complementaria del éxito de la acción instrumental, es decir, la posibilidad de un acuerdo sin coerciones y de reconocimiento sin violencia» ${ }^{65}$.

Habermas, recurriendo a la crítica pragmatista de Peirce contra el objetivismo de la ciencia natural y a la crítica historicista contra el objetivismo de las ciencias del espíritu de Dilthey, expone una discusión inmanente del presupuesto positivista según el cual la ciencia refleja un en sí de hechos legalmente estructurados al margen de una constitución previa del sujeto. En efecto, si ocultamos al sujeto constituyente no reconocemos la acción comunicativa en la que sujetos copartícipes en situaciones de diálogo rebasan las categorías generales del lenguaje cuando expresan su propia subjetividad individual. Y si no reconocemos la necesidad de mejorar la praxis vital comunicativa para generar consensos sin coacciones, entonces anulamos la especificidad de las ciencias hermenéuticas.

En la tercera parte del libro ${ }^{66}$, cuando ya se ha demostrado la diferencia entre ciencias hermenéuticas y ciencias empírico-analíticas, puede ya Habermas presentar, con todas las credenciales, el interés técnico y el interés práctico como intereses diversificados del conocimiento. El punto de partida de Habermas es el sujeto constituyente, pero no el sujeto trascendental de Kant. Su lugar es ocupado por una especie que se constituye a sí misma, tanto mediante la actividad técnico-instrumental como de la actividad comunicativa. Ambas actividades, trabajo e interacción, en contextos vitales fácticos, son las condiciones fundamentales para la reproducción y supervivencia de la especie. Por este motivo, son el origen del que provienen los marcos categoriales de las dos orientaciones básicas de la investigación: la ciencia empírica y la ciencia hermenéutica. Ambas ciencias encuentran sus condiciones de validez en que rinden y funcionan con vistas a los dos grandes intereses de la especie imprescindibles para su reproducción y supervivencia; el interés técnico para la manipulación y dominio de la naturaleza y el interés práctico para una comprensión entre sujetos que genere consensos sin violencia.

Nos queda por último el interés emancipatorio. El procedimiento de prueba es exactamente el mismo. Dada la existencia del psicoanálisis freudiano como ciencia de la autorreflexión crítica que pretende que el sujeto sea consciente de todos aquellos aspectos de su identidad que se sustraen a la comunicación pública, es decir, rememorar todos aquellos conflictos irresueltos que nos obligan a reprimir necesidades y que al imprimir un límite interior en el sujeto fragmentan su identidad, entonces ha de existir el interés cognoscitivo emancipatorio como tercer interés del conocimiento. Este tercer interés reside en la "fuerza emancipatoria de la reflexión que el sujeto verifica en sí en la medida en que se hace transparente a sí mismo en su propia historia genética ${ }^{67}$. La autorreflexión crítica no tiene como condición necesidades que provengan de contextos vitales fácticos, sino que «en la autorreflexión, un conocimiento por mor del conocimiento coincide con el interés por la emancipación; pues la realización de la reflexión se sabe como movimiento de la emancipación ${ }^{68}$. En efecto, la autorreflexión crítica equivale a

\footnotetext{
64 Cfr. Habermas, J., CI, p. 183/ EuI, p. 221.

65 Cfr. Habermas, J., CI, p. 183/ EuI, pp. 221-222.

66 Cfr. Habermas, J., CI, p. 245-270/EuI, pp. 262-300. Consultar el apartado Autorreflexión como ciencia.

67 Cfr. Habermas, J., CI, p. 200/ EuI, pp. 243-244.

68 Cfr. Habermas, J., CI, p. 201/ EuI, p. 244.
} 
emancipación y por ello es ella misma su propio interés. En el párrafo anteriormente citado dice Habermas

la razón está bajo el interés por la razón...Podemos decir que sigue un interés cognoscitivo emancipatorio que tiene como meta la realización de la autorreflexión como tal.

Sin embargo, en realidad, no se trata de un tercer interés en el mismo sentido que los otros dos. En una interesante cita nos explica Habermas:

Pues es verdad que más bien ocurre que la categoría de interés cognoscitivo viene testificada por el interés innato a la razón. Sólo a partir de su conexión con el interés cognoscitivo emancipatorio de la reflexión racional pueden los intereses técnico y práctico ser comprendidos sin malentendidos como intereses rectores del conocimiento ${ }^{69}$.

Por lo tanto, el interés cognoscitivo emancipatorio es denominado por Habermas como un interés innato a la razón, en lo que se diferencia de los otros dos intereses. En efecto, citando a Kant, nos dice Habermas que interés, en general, es «la satisfacción que vinculamos a la representación de la existencia de un objeto o de una acción ${ }^{70}$. Y nos representamos la existencia de objetos, ya sea en el marco de las ciencias empíriconomológicas en las que se «aprehende la realidad con vistas a una manipulación técnica posible siempre y en cualquier parte bajo condiciones específicas ${ }^{71} \mathrm{O}$, en otro contexto de aplicación, en el marco de las ciencias hermenéuticas donde se «aprehenden interpretaciones de la realidad con vistas a la intersubjetividad posible de un acuerdo orientador de la acción ${ }^{72}$, porque rinden y son efectivos para una "especie que se reproduce a sí misma bajo condiciones culturales, es decir, una especie que sólo se constituye a sí misma en un proceso de formación ${ }^{73}$. En esto, se ve claramente en qué difieren el interés por la actividad técnica instrumental y por la actividad comunicativa del interés emancipatorio; los dos primeros no corresponden a un ser racional puro descontextualizado sino que «son orientaciones básicas que son inherentes a determinadas condiciones fundamentales de la reproducción y autoconstitución posibles de la especie humana ${ }^{74}$. Interés cognoscitivo técnico y práctico como «intereses rectores del conocimiento se miden sólo en aquellos problemas de la conservación de la vida ${ }^{75}$, aunque la reproducción y conservación biológica de la vida se encuentra desde el principio bajo condiciones culturales. En cambio el interés cognoscitivo emancipatorio, a diferencia de los otros dos intereses, no está restringido a contextos vitales fácticos, a saber, que la reproducción de la vida a nivel antropológico esté determinada culturalmente por el trabajo y la interacción, sino que efectúa una autorreflexión crítica en la que el sujeto se hace transparente a sí mismo cuya finalidad, más allá de la mera autofundamentación de la razón, es "promover la autorreflexión del enfermo bajo las condiciones de una compulsión y el correspondiente interés por suprimir esa compulsión ${ }^{76}$.

Por lo tanto, la autorreflexión crítica no se queda sólo en un plano meramente lógico, sino que tiene como condiciones previas la presión del sufrimiento debido a la represión excedente y el interés del sujeto por su propia emancipación.

69 Cfr. Habermas, J., CI, p. 201/ EuI, p. 244.

70 Cfr. Habermas, J., CI, p. 201/ EuI, p. 244.

71 Cfr. Habermas, J., CI, p. 198/ EuI, p. 241

72 Cfr. Habermas, J., CI, p. 199/ EuI, p. 241.

73 Cfr. Habermas, J., CI, p. 198/ EuI, p. 240.

74 Cfr. Habermas, J., CI, p. 199/ EuI, p. 242.

75 Cfr. Habermas, J., CI, p. 199/ EuI, p. 242.

76 Cfr. Habermas, J., CI, p. 283/ EuI, p. 349. 
La autonomía y la emancipación como interés del conocimiento son inmanentes a la actividad autorreflexiva y crítica del sujeto; inmanentes, es decir, que el interés por la emancipación es lo mismo que la autorreflexión crítica y que por ello, en este punto, conocimiento e interés están fusionados en acto. Que la emancipación es lo mismo que autorreflexión crítica significa que el proceso por el cual el sujeto se hace transparente a sí mismo es imposible sin una voluntad o interés de éste por su propia emancipación. Recurriendo al psicoanálisis nos dice Habermas sobre lo anterior

La crítica no tendría el poder de vencer la falsa conciencia si no fuese impulsada por una pasión por la crítica. O de una parte de la experiencia de dolor y de la necesidad y del interés por la superación de esta situación opresiva....la presión del sufrimiento y el interés en la curación no son sólo ocasión para el inicio de la terapia, sino un presupuesto para el éxito de la misma.

La terapia psicoanalítica...su éxito depende....de la autorreflexión del enfermo. Pero ésta sólo sigue en marcha en la medida en que el interés por el autoconocimiento sigue empujando al conocimiento analítico contra las resistencias motivacionales ${ }^{77}$.

En la segunda parte de la cita se dice que sólo a partir de su conexión con el interés cognoscitivo emancipatorio de la reflexión racional pueden los intereses técnico y práctico ser comprendidos sin malentendidos como intereses rectores del conocimiento. En efecto, un interés es rector del conocimiento cuando es inmanente a la actividad racional y no está meramente condicionado por una circunstancia externa para su ejercicio. Tanto el interés técnico, cuyo objetivo es el sometimiento de la naturaleza, como el interés práctico, por el que se organizan las relaciones de los hombres entre sí, constituyen lo que Freud entiende por cultura, es decir, «aquello por lo cual la especie humana se eleva sobre las condiciones de la existencia animal ${ }^{78}$. En el seno de la cultura ambas funciones no son independientes sino que la relación entre sí de los hombres depende de los bienes disponibles, y a su vez estos dependen del grado de desarrollo técnico de una cultura. De este modo, cuanto mayor sea el grado de desarrollo del poder técnico, menor será el grado de represión necesaria sobre el potencial pulsional excedente del individuo para la autoconservación del sistema social. Esto no significa conceder prioridad al proceso de producción, sólo que el desarrollo de éste abre la posibilidad interna para reducir el grado de represión necesaria sobre el individuo:

La presión de la realidad y la represión que socialmente le corresponde dependen tanto del grado de desarrollo técnico frente a las fuerzas de la naturaleza como de los modos en los que se organiza su explotación y la distribución de los bienes producidos. Cuanto más se amplíe el poder de los recursos técnicos y se dulcifique la presión de la realidad, tanto más débil será la censura de los impulsos impuesta al sistema de autoconservación y tanto más fuerte será la organización del yo y su capacidad de dominar de una manera racional las renuncias ${ }^{79}$.

Además, el límite sobre lo que es compatible e incompatible con la realidad social es variable, ya que «el grado de represión socialmente necesaria se mide en el alcance variable del poder de disposición técnica sobre los procesos naturales» ${ }^{80}$.

Por este motivo, ni el interés técnico ni el práctico, ni la cultura en general son inmanentes a la razón porque por sí mismas no emancipan al individuo, es más, el sistema social en su totalidad podría ser en sí mismo patológico y ello ocurre cuando los

77 Cfr. Habermas, J., CI, p. 234/ EuI, pp. 286-287.

78 Cfr. Habermas, J., CI, p. 273/ EuI, p. 336.

79 Cfr. Habermas, J., CI, p. 272/ EuI, pp. 334-335.

80 Cfr. Habermas, J., CI, p. 276/ EuI, p. 339. 
niveles de represión en una sociedad son excedentes. Cuando la represión es excedente se le exigen al individuo conductas uniformes innecesarias que se repiten rígidamente y sin crítica, por lo que el individuo no puede racionalizar las renuncias a sus pulsiones internas que le impone el sistema de autoconservación social. De este modo, surgen satisfacciones virtuales de los deseos reprimidos que Freud denomina ilusiones. En éstas encuentra una satisfacción sustitutiva todo aquello que es incompatible con la realidad social. Las ilusiones en sí mismas no suponen una fuga evasiva del yo en contradicción directa con la realidad, ya que aquellas pueden tener un contenido utópico. Al contrario, se pueden volver delirantes cuando dichas satisfacciones sustitutivas, al hacerse inmunes a toda crítica, se repiten compulsivamente y sirven para legitimar la dominación por encima del nivel exigido.

El único interés que es rector del conocimiento, porque está fusionado en acto con la autoclarificación crítica, es el interés emancipatorio. Utilizando el lenguaje aristotélico sería lo único que se busca por sí mismo. Sobre esta base y recordando a Freud comenta Habermas:

El desarrollo de las fuerzas productivas crea en cada nivel la posibilidad objetiva de suavizar la violencia del marco institucional y la de sustituir la base afectiva de obediencia a la cultura por una base racional.

La meta es la fundamentación racional de los preceptos de la cultura, es decir, la organización de las relaciones sociales según el principio de que el valor de toda norma que implique consecuencias políticas ha de depender de un consenso brotado de una comunicación libre de violencia ${ }^{81}$.

La finalidad última de toda cultura, o mejor dicho, su «esperanza racional» ${ }^{82}$, es que el poder técnico crezca lo suficiente para que se pueda dar la posibilidad objetiva de una organización social en la que no exista represión excedente. Y si ésta no se da, entonces los individuos pueden asumir los costos represivos del orden social sin necesidad de satisfacciones virtuales, sino sólo con una fundamentación racional de las normas sociales. Respecto a una sociedad en la que sus individuos sean capaces de asumir racionalmente la represión necesaria para la autoconservación del sistema social sin recurrir a una satisfacción de los motivos reprimidos mediante las fantasías del deseo, no tenemos ninguna anticipación totalitaria de sentido que nos garantice la seguridad de su consecución. Esta expectativa es irracional y se trata sólo de una ilusión delirante provocada por la represión excedente sobre los individuos. En efecto, para Habermas

esta esperanza racional es lo que separa radicalmente la intención de la Ilustración de las tradiciones dogmáticas.....Esta precaución no impide la actividad crítico-revolucionaria, pero sí la certeza totalitaria de que la idea por la que esa actividad se deja guiar con muy buenas razones, sea realizable en todas las circunstancias ${ }^{83}$.

Así pues, sólo tenemos expectativas refutables en las que «el interés de la razón tiende hacia una realización crítico-revolucionaria progresiva, pero a modo de ensayo, de las grandes ilusiones humanas, en las que los motivos reprimidos han sido reelaborados y convertidos en fantasías de la esperanza» ${ }^{84}$.

Reproducimos una extensa cita en la que Habermas insiste en el interés emancipatorio como verdadero interés rector del conocimiento:

81 Cfr. Habermas, J., CI, p. 279/ EuI, p. 344.

82 Cfr. Habermas, J., CI, p. 280/ EuI, p. 345.

83 Cfr. Habermas, J., CI, p. 280/ EuI, p. 345.

84 Cfr. Habermas, J., CI, p. 284/ EuI, p. 350. 
El interés por la autoconservación no puede tener como meta, así sin más, la reproducción en la vida de la especie, ya que esa especie, bajo las condiciones culturales de la existencia, tiene que empezar interpretando qué es lo que se entiende por vida. Estas interpretaciones se orientan, a su vez, según las ideas de la vida buena....para satisfacer y dar articulación a un interés fundamental: el interés por la medida de la emancipación que históricamente es objetivamente posible ${ }^{85}$.

Las interpretaciones acerca de la vida buena no son ni una mera convención arbitraria ni una esencia inmutable sobre la naturaleza humana, sino algo que se fantasea cuando el potencial pulsional primario excedente rebasa las posibilidades de satisfacción inmediata. En efecto, el ser humano es «el animal que inhibe sus impulsos y a la vez fantasea» ${ }^{86}$, pero dichos ideales sobre la vida buena "tienen que estar fantaseados con la suficiente exactitud como para satisfacer y dar articulación a un interés fundamental: el interés por la medida de la emancipación ${ }^{87}$. Y un poco más abajo en la misma página nos dice:

Mientras los hombres hayan de conservar su vida por el trabajo y la interacción, renunciando a sus impulsos, es decir, bajo la compulsión de una comunicación distorsionada, el interés por la autoconservación tiene necesariamente la forma del interés de la razón, que sólo se despliega en la crítica y se confirma en las consecuencias prácticas de ésta.

IES Salvador Serrano

Eduardo CONTRERAS

Alcaudete, Jaén

eduardocont1@hotmail.com

[Artículo aprobado para su publicación en noviembre de 2013]

85 Cfr. Habermas, J., CI, p. 284/ EuI, p. 350.

86 Cfr. Habermas, J., CI, p. 278/ EuI, p. 343.

87 Cfr. Habermas, J., CI, p. 284/ EuI, p. 350. 